\title{
Investigating the relative efficacies of combination chemotherapy of paclitaxel/carboplatin, with or without anthracycline, for endometrial carcinoma
}

\author{
Kazuko Fujiwara $\cdot$ Tomomi Egawa-Takata $\cdot$ Yutaka Ueda $\cdot$ Toshihiro Kimura \\ Kiyoshi Yoshino $\cdot$ Masami Fujita $\cdot$ Takashi Miyatake $\cdot$ Yukinobu Ohta $\cdot$ \\ Shoji Kamiura · Takayuki Enomoto · Tadashi Kimura
}

Received: 20 April 2011 / Accepted: 8 July 2011 / Published online: 30 November 2011

(C) The Author(s) 2011. This article is published with open access at Springerlink.com

\begin{abstract}
Purpose Recently a combination of paclitaxel and carboplatin (TC) (without an anthracycline) has begun to be used as an adjuvant or remission induction therapy, without any critical supportive evidence of its efficacy relative to a combination chemotherapy of taxane, platinum and anthracycline such as TEC (paclitaxel, epirubicin and carboplatin). The aim of our present study was to conduct the required clinical evaluations of the relative effectiveness of TC compared to TEC.

Methods A retrospective comparison between the efficacy of TEC and TC regimens used for endometrial carcinoma at the Osaka University Hospital and the Osaka Medical Center for Cancer and Cardiovascular Diseases in Osaka, Japan, respectively, from 1999 to 2009 was performed. The clinical characteristics of the patients who received either TEC or TC were not significantly different, and TEC and TC therapies were initiated based on similar indications for chemotherapy. TEC regimen was paclitaxel $\left(150 \mathrm{mg} / \mathrm{m}^{2}\right)$,
\end{abstract}

K. Fujiwara and T. Egawa-Takata contributed equally to this work

K. Fujiwara $\cdot$ T. Miyatake $\cdot$ Y. Ohta $\cdot$ S. Kamiura Department of Gynecology, Osaka Medical Center for Cancer and Cardiovascular Diseases, 1179-3, Higashinari-ku-Nakamichi, Osaka 537-8511, Japan

T. Egawa-Takata

Department of Obstetrics and Gynecology, Osaka Police Hospital, 10-31, Kitayama-cho, Tennoji-ku, Osaka 543-0035, Japan

Y. Ueda $(\bowtie) \cdot$ T. Kimura $\cdot$ K. Yoshino $\cdot$ M. Fujita $\cdot$ T. Enomoto

T. Kimura

Department of Obstetrics and Gynecology,

Osaka University Graduate School of Medicine,

2-2, Yamadaoka, Suita, Osaka 565-0871, Japan

e-mail: ZVF03563@nifty.ne.jp epirubicin $\left(50 \mathrm{mg} / \mathrm{m}^{2}\right)$ and carboplatin (AUC 4). TC regimen consisted of paclitaxel $\left(175 \mathrm{mg} / \mathrm{m}^{2}\right)$ and carboplatin (AUC 5).

Results TEC was demonstrated to provide significantly better survival than TC as an adjuvant therapy for resected Stage III/IV diseases $(p=0.017$ for progression-free survival and $p=0.014$ for overall survival, by the log-rank test). However, in recurrent or more advanced cases, TC and TEC demonstrated similar effects on survival $(p=0.55$ for progression-free survival and $p=0.63$ for overall survival).

Conclusions TEC should be offered as an adjuvant therapy to Stage III/IV patients. TC may be considered for recurrent or unresectable cases as a remission induction therapy.

Keywords Endometrial carcinoma $\cdot$ Platinum $\cdot$ Taxane . Anthracycline $\cdot$ Survival

$\begin{array}{ll}\begin{array}{l}\text { Abbreviations } \\ \text { TAP or AP }\end{array} & \begin{array}{l}\text { Doxorubicin and cisplatin } \\ \text { (with or without paclitaxel) }\end{array} \\ \text { TC or TEC } & \begin{array}{l}\text { Paclitaxel and carboplatin } \\ \text { (without or with epirubicin) }\end{array} \\ \text { TEP } & \text { Paclitaxel, epirubicin and cisplatin } \\ \text { ALT } & \text { Alanine aminotransferase } \\ \text { AST } & \text { Aspartate aminotransferase } \\ \text { AUC } & \text { Area under the plasma drug concentration } \\ & \text { versus time curve } \\ \text { CR } & \text { Complete response } \\ \text { G-CSF } & \text { Granulocyte-colony stimulating factor } \\ \text { GOG } & \text { Gynecologic Oncology Group } \\ 5-H T 3 & \text { 5-Hydroxytriptamine-3 } \\ \text { JGOG } & \text { Japanese Gynecologic Oncology Group } \\ \text { OS } & \text { Overall survival }\end{array}$


PD Progressive disease

PFS Progression-free survival

PR Partial response

SD Stable disease

\section{Introduction}

Endometrial cancer is the most common gynecological cancer in the United States, and its incidence has increased significantly during the last three decades. Current surgical endometrial cancer therapy consists of a hysterectomy, bilateral salpingo-oophorectomy and retroperitoneal lymph node dissection $[1,2]$. A randomized study by the Gynecologic Oncology Group (GOG) revealed that a combination chemotherapy of AP (doxorubicin and cisplatin) was superior to the traditional whole abdominal irradiation as an adjuvant therapy (GOG \#122). Unfortunately, significant hematological and cardiac toxicity and treatment-related death was soon associated with AP treatments [3].

Platinum and anthracycline have long been used as the gold standard drugs for advanced or recurrent endometrial carcinomas [4, 5]. Recently, taxane has been added to this group [6, 7]. A recent study showed better survival following a TAP therapy (paclitaxel, doxorubicin and cisplatin) than for AP (GOG \#177) [8]; however, neurological toxicity was even greater for patients receiving TAP, with $39 \%$ of the patients suffering Grade 2-3 peripheral neuropathy.

Lissoni et al. [9] reported that TEP (paclitaxel, epirubicin and cisplatin) exhibited superior anti-tumor activity against advanced endometrial carcinoma. Recently, TEC (paclitaxel, epirubicin and carboplatin) was shown to have improved activity against metastatic and recurrent endometrial carcinomas, and was found to be relatively tolerable when given with G-CSF support [10]. In our own recent phase I/II prospective studies of TEC, we analyzed the optimal dose for TEC therapy of our Japanese population, which we subsequently determined to be $150 \mathrm{mg} / \mathrm{m}^{2}$ paclitaxel, $50 \mathrm{mg} / \mathrm{m}^{2}$ epirubicin, and AUC 4 carboplatin Takata et al. [18]. Based on these findings, TEC has become our new standard for endometrial carcinoma treatment. Recently, however, TC (paclitaxel and carboplatin) has been begun to be widely applied for treatment of endometrial carcinoma, based on its initial reported effectiveness and high tolerability [11-13]. However, its equivalency or superiority to TEC has never been rigorously demonstrated, thus TC therapy is not currently an established regimen for endometrial carcinoma.

Gynecologic Oncology Group (GOG) has an ongoing study (\#209) to compare TC with TAP, and the Japanese Gynecologic Oncology Group (JGOG) is performing a similar prospective study (JGOG \#2043) to compare three combination chemotherapies: TC, DP (docetaxel and cisplatin) and AP. It has been of great interest to gynecologists whether TC alone is a sufficient chemotherapy for endometrial carcinoma or whether anthracycline is additionally required.

In our present study, we performed a retrospective comparison of TEC versus TC against endometrial carcinoma. We compared the patients' data for TEC therapy, which was exclusively performed at the Osaka University Hospital as part of our phase II study of TEC therapy (submitted), with those of TC, which was administered at our sister Osaka Medical Center for Cancer and Cardiovascular Diseases.

\section{Materials and methods}

A retrospective comparison was conducted between the relative efficacies of the TEC and TC regimens, which were performed for endometrial carcinoma cases at the Osaka University Hospital and the Osaka Medical Center from 1999 to 2009. During this period, TEC therapy was exclusively used at the Osaka University Hospital for all its endometrial carcinoma cases with indications for chemotherapy. A TEC dosage of $150 \mathrm{mg} / \mathrm{m}^{2}$ for paclitaxel, $50 \mathrm{mg} / \mathrm{m}^{2}$ for epirubicin and AUC 4 for carboplatin was used, based on our phase I results with a Japanese population (submitted). On the other hand, at the Osaka Medical Center, a TC regimen of paclitaxel $175 \mathrm{mg} / \mathrm{m}^{2}$ and carboplatin AUC 5 (based on the results of our phase I study for ovarian carcinoma in a Japanese population, preliminarily reported by Ueno et al. [14]) was used instead of TEC for all their endometrial carcinoma cases with indications for chemotherapy. The gynecologic surgeons who performed the surgical treatments were all trained at the Osaka University Hospital, and the surgical procedures, and the indications for pelvic and para-aortic lymph node dissection, were identical in the two hospitals. Moreover, adjuvant chemotherapy was performed using the similar indicators.

Eligibility for TEC and TC chemotherapies required that the patient have adequate findings in the following: hematology (WBC $\geq 3,000 / \mu \mathrm{l}$, platelets $\geq 100,000 / \mu \mathrm{l}$, granulocytes $\geq 1,500 / \mu \mathrm{l}$ and hemoglobin $\geq 10 \mathrm{~g} / \mathrm{dl}$ ), renal (creatinine $\geq 2 \mathrm{mg} / \mathrm{dl}$ ) and hepatic [bilirubin $\geq 3 \mathrm{mg} / \mathrm{dl}$, aspartate aminotransferase (AST) and alanine aminotransferase (ALT) $\geq 2$ times the international normal value]. A relative performance status of 0-2 was needed. The tumors needed to be histopathologically diagnosed as being either a primary or recurrent endometrial carcinoma. In the current study, the clinicopathological features of the cases in which TEC or TC chemotherapy were performed, including the age of the patient, the histology and the stage of the disease, and the adverse effects of each chemotherapy regimen were retrospectively reviewed utilizing their clinical records, 
including physical examination notes, radiological reports, operative records, and histopathology reports. The histological diagnoses were made by authorized pathologists from the Departments of Pathology of the Osaka University and the Osaka Medical Center, who were all trained at the Osaka University Hospital.

In order to evaluate the efficacy of TEC and TC chemotherapies against endometrial carcinoma, progression-free survival (PFS) and overall survival (OS) were calculated. PFS was measured from the administration of chemotherapy to the date of the radiologic or pathologic diagnosis of relapse, or to the date of the last follow-up. OS was defined as the period from the start of chemotherapy to the patient's disease-specific death or to the date of the last follow-up. In order to evaluate the anti-tumor effect of TEC and TC chemotherapies against the advanced diseases which were unresectable by surgery and the recurrent diseases, previously described standard criteria from the World Health Organization [15] and Pectasides et al. [16] were used. The tumors were assessed by CT scan and/or MRI at baseline and every three treatment courses thereafter. A complete response (CR) was defined as the disappearance of all known disease, determined by two observations not less than 4 weeks apart. We used RECIST (Response Evaluation Criteria in Solid Tumors, version 1.0) for evaluating the therapy response. ACR required regression of all tumors. A partial response (PR) required $>30 \%$ reduction in the largest diameter of the largest lesion. A progressive disease (PD) was defined as one in which new lesions appeared, or the largest diameter of the largest lesion enlarged more than $20 \%$. All others were considered to be stable disease (SD).

Adverse treatment effects were also analyzed. They were graded based on the National Cancer Institute's Common Toxicity Criteria (version 2.0). Granulocyte-colony stimulating factor (G-CSF) was administered to improve immune function whenever the total $\mathrm{WBC} /$ neutrophil count decreased to under $1,000 / 500$ per $\mu \mathrm{l}$, or when febrile neutropenia was observed. A histamine $\mathrm{H} 15-\mathrm{HT}_{3}$ antagonist was administrated orally before the paclitaxel to prevent both emesis and an allergic reaction. Other antiemetic drugs were administered as needed.

A regimen of either TEC or TC was administered every 3-4 weeks for 3 weeks against resected Stage I/II diseases with risk factors, which included a myometrium invasion depth of $>1 / 2$ and or an atypical histology (such as endometrioid adenocarcinoma Grade 3, clear cell carcinoma or serous papillary carcinoma). TEC or TC was given as an adjuvant therapy for 6 weeks against resected Stage III/IV diseases and unresectable or recurrent diseases. The cases which were classified as Stage IIIa due to positive peritoneal cytology alone, without any other risk factors, were excluded from as having an indication for adjuvant chemo- therapy. In order to compare the efficacy of TEC and TC regimes accurately, all the cases in which these chemotherapies were attempted were included in this analysis, including the cases in which these chemotherapies were canceled underway due to severe toxicities or to the patient's intermittent desire to stop chemotherapy.

The Osaka University Hospital protocol for TEC administration was to be given to only patients who were 70 years of age or less; the Osaka Medical Center protocol for TC allowed few patients who were over 70 years of age. The present comparative analysis was conducted only for those patients who were 70 years of age or less. In all cases, chemotherapy was performed only in those patients who were expected to have an estimated remaining survival of greater than 3 months.

\section{Statistical analysis}

MedCalc (MedCalc Software, Mariakerke, Belgium) was used for the statistical analyses. The distribution of patients' age, tumor histology and stage were analyzed by the MannWhitney $U$-test, the Chi-square test, or Fisher's exact test. PFS and OS curves were constructed using the KaplanMeier method and were evaluated for statistical significance by the log-rank test. The frequency of adverse effects in the two groups and the response of each chemotherapy were compared by Fisher's exact test. Results were considered to be significant when the $p$ value was less than 0.05 .

\section{Results}

Clinical characteristics of the patients with unresectable or recurrent disease who received TEC or TC chemotherapy as a remission induction therapy

TEC therapy was intended for 28 patients with unresectable or recurrent disease at the Osaka University Hospital, and TC therapy was attempted in 23 patients with similar diseases at the Osaka Medical Center. Distributions of age, histology and disease status did not exhibit any significant differences between the TEC and the TC groups (Table 1).

In the recurrent cases, all the patients underwent surgical treatment as a first treatment. The initial tumor stage in the TEC group was Stage I in three cases, II in one case and III in six cases; in the TC group it was I in five cases, II in two cases, III in three cases and IV in one ( $p=0.42$ by the Chisquare test). Adjuvant chemotherapy or radiation had been performed postoperatively in $8(80 \%)$ of 10 cases in TEC group and $8(73 \%)$ of 11 cases in TC group. TEC or TC was administered as the first treatment in all the recurrent disease cases. In advanced cases with unresectable disease, 7 cases were at Stage III and 11 cases were in Stage IV in 
Table 1 Clinical characteristics of the advanced and recurrent cases in which TEC or TC chemotherapy was performed as a remission induction therapy

\begin{tabular}{llcl}
\hline Characteristic & TEC $(n=28)$ & TC $(n=23)$ & $p$ value \\
\hline Age & $57(34-69)$ & $56(32-70)$ & 0.95 \\
Histology & & & 0.54 \\
$\quad$ Endometrioid & $10(36 \%)$ & $7(30 \%)$ & \\
$\quad$ Non-endometrioid & $18(64 \%)$ & $16(70 \%)$ & \\
Disease status & & & 0.41 \\
$\quad$ Advanced & $18(64 \%)$ & $12(52 \%)$ & \\
Recurrent & $10(36 \%)$ & $11(48 \%)$ & \\
\hline
\end{tabular}

Clinical characteristics of the primary endometrial carcinoma cases with unresectable diseases and the recurrent cases are shown. Distributions of age, histology and disease status did not exhibit any significant differences between the TEC and TC groups

the TEC group, and 5 cases were in Stage III and 7 cases were in Stage IV in the TC group, demonstrating no significant difference ( $p=0.88$ by Fisher's exact test).

Anti-tumor effect of TEC and TC therapies in the patients with unresectable or recurrent disease

Complete response (CR) or PR was achieved in 14 of 18 (78\%) and 6 of $12(50 \%)$ advanced cases by TEC and TC, respectively ( $p=0.11$ by Fisher's exact test), and 5 of 10 (50\%) and 6 of $11(55 \%)$ recurrent cases by TEC and TC, respectively ( $p=0.83$ by Fisher's exact test) (Table 2). In total, the response rate of TEC therapy against recurrent or advanced diseases was $68 \%$ (19 of 28 cases), and that of TC was $52 \%$ ( 12 of 23 cases). This $16 \%$ better difference in response rates between TEC and TC therapies was not statistically significant ( $p=0.25$ by Fisher's exact test).

Survival effect of TEC and TC therapies in the patients with unresectable or recurrent disease

Both OS and PFS did not demonstrate any significant difference between the TEC and TC groups $(p=0.63$ for

Table 2 Anti-tumor effect (response rate) of TEC and TC chemotherapies

\begin{tabular}{lcc}
\hline Response to chemotherapy & TEC $(n=28)$ & TC $(n=23)$ \\
\hline CR/PR & $19(68 \%)$ & $12(52 \%)$ \\
SD/PD & $9(32 \%)$ & $11(48 \%)$ \\
\hline
\end{tabular}

The anti-tumor effect of TEC and TC was evaluated in advanced or recurrent cases. Response rates were $68 \%$ in the TEC group and $52 \%$ in the TC group. This difference was not statistically significant

$C R$ complete response, $P R$ partial response, $S D$ stable disease, $P D$ progressive disease

$p=0.25$ (Fisher's exact test)
OS and $p=0.55$ for PFS, by the log-rank test) (Fig. 1). By analyzing the effect of TEC and TC on survival in subgroups of recurrent cases and advanced (unresectable) cases, TEC therapy was demonstrated to be relatively more effective than TC in unresectable cases $(p=0.17$ and $p=0.75$ for PFS and OS, respectively). TC therapy, on the other hand, provided a better survival effect than TEC in recurrent cases $(p=0.32$ and $p=0.22$ for PFS and OS, respectively), however these differences were not statistically significant.

Clinical characteristics of the completely resected patients in Stage III/IV who received TEC or TC chemotherapy as an adjuvant therapy

Because in our previous study (submitted for publication), we could not demonstrate a significant survival improvement in Stage I/II cases using TEC compared to radiation as the adjuvant therapy, and because the effects of TEC and TC therapy on survival were not different significantly in our present study, a comparison analysis of the survival effects of TEC and TC was focused completely on resected Stage III and IV cases.

TEC was administered to 47 patients at the Osaka University Hospital, and TC to 30 patients at the Osaka Medical Center. Their clinical features are shown in Table 3. Distributions of age, histology and stage did not exhibit any significant difference between the TEC and the TC groups. The cases which were classified as being in Stage IIIa due to positive peritoneal cytology alone (without any other risk factors) were excluded from adjuvant chemotherapy, and thus this study.

Survival effect of adjuvant TEC and TC therapies in the completely resected patients in Stage III/IV

The OS and PFS curves of the TEC and TC groups were shown in Fig. 2. The median follow-up period was 38 months (2-105 months). PFS exhibited a statistically significant difference between the TEC and TC groups ( $p=0.017$ by the log-rank test, Hazard Ratio: $0.3838 ; 95 \%$ CI: 0.1709-0.8623). Moreover, OS also exhibited a statistically significant difference between TEC and TC groups ( $p=0.014$ by the log-rank test, Hazard Ratio: $0.3108 ; 95 \%$ CI: 0.1048-0.9214). Thus, TEC therapy was demonstrated to provide a significant improvement of survival as an adjuvant therapy for resected Stage III/IV diseases.

\section{Adverse effects of TEC and TC therapies}

Adverse effects of the TEC and TC chemotherapies were evaluated in 187 patients whose accurate data were available (Table 4).Hematological toxicity tended to be more 


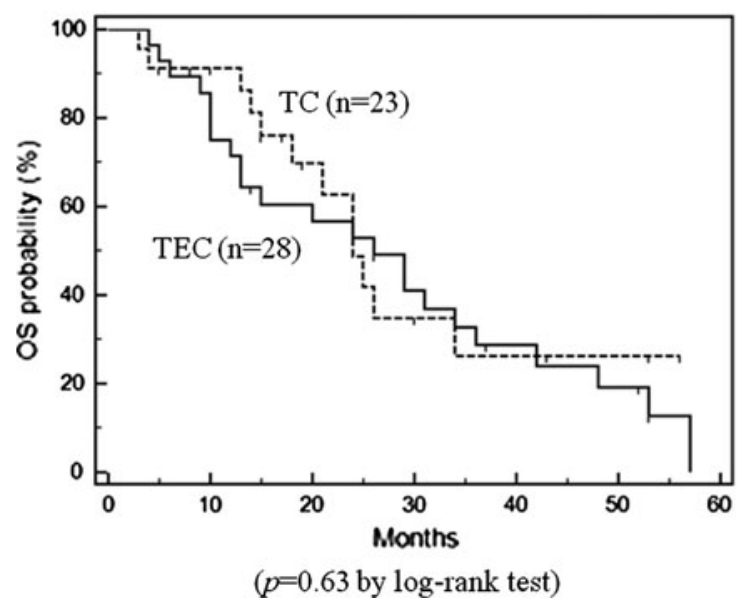

Fig. 1 PFS and OS of TEC and TC groups for unresectable or recurrent diseases. Both PFS and OS were not demonstrated to be significantly different between the TEC and TC groups ( $p=0.63$ and $p=0.55$

Table 3 Clinical characteristics of the completely resected Stages III and IV patients to whom TEC or TC chemotherapy was performed as an adjuvant therapy

\begin{tabular}{lccc}
\hline Characteristic & TEC $(n=47)$ & TC $(n=30)$ & $p$ value \\
\hline Age & $56(3-69)$ & $59(34-70)$ & 0.23 \\
Histology & & & 0.54 \\
Endometrioid & $33(70 \%)$ & $23(77 \%)$ & \\
Non-endometrioid & $14(30 \%)$ & $7(23 \%)$ & \\
Stage & & & 0.83 \\
IIIa & $14(30 \%)$ & $8(27 \%)$ & \\
IIIb & $1(2 \%)$ & $1(3 \%)$ & \\
IIIc & $26(55 \%)$ & $15(50 \%)$ & \\
IVb & $6(13 \%)$ & $6(20 \%)$ & \\
\hline
\end{tabular}

The endometrial carcinomas, confined to the uterus, which were classified in Stage IIIa based on only positive peritoneal cytology, were excluded. Distributions of age, histology and stage did not exhibit any significant differences between the TEC and TC groups

frequently observed in the TEC group than in the TC group $(p=0.065)$; however, a statistical significance was not detected. Non-hematological toxicity was observed to be similar in the TEC and TC groups $(p=0.49)$. The nonaccomplishment rates of the scheduled TEC and TC therapies (due to severe toxicities) were not different between the two regimens $(p=0.81)$. Those due to the patients' intermittent desire to stop chemotherapy were also not statistically different between the two regimens $(p=0.37)$.

\section{Conclusions}

Adjuvant chemotherapy for endometrial carcinoma is one of the hottest topics in gynecologic oncology. Platinum, anthracycline and taxane derivatives are regarded as critical

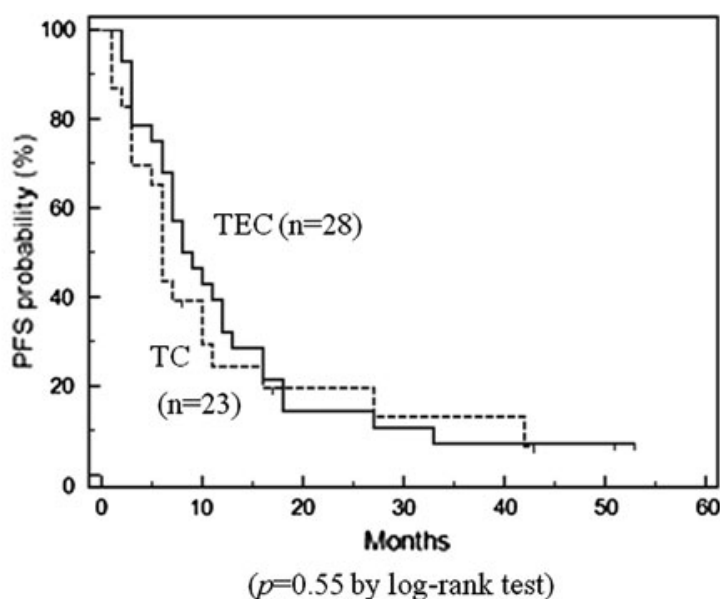

by log-rank test, respectively). Solid line TEC group. Broken line TC group

drugs for treatment of advanced or recurrent endometrial carcinomas [4, 6, 7]. Although combination chemotherapies using these drugs, such as TAP, TEP and TEC, are effective, moderate-to-severe side effects are sometimes observed by Fleming et al. [8], Lissoni et al. [9], Papadimitriou et al. [10] and our previous study (Takata et al. [18]).

TC, which uses only paclitaxel and carboplatin, is the current gold standard therapy for ovarian carcinoma due to its high effectiveness coupled with its high tolerability [17]. TC chemotherapy was somewhat effective for endometrial carcinoma [11-13]. It is, therefore, easy to understand why TC, although not yet an established standard therapy, has been frequently applied worldwide for the treatment of endometrial carcinoma cases. However, until this current work, a comparison of the usefulness of TC to other combination chemotherapies using these three key drugs, such as TAP, TEP and TEC, has not been reported.

A GOG study to compare the combination of TC with TAP (GOG \#209) has finished recruitment of the patients; however, analysis of the survival effects of the two regimens may require a few years; a similar JGOG study to compare the combination chemotherapies TC, DP and AP (JGOG \#2043) is even less far along; it is still under registration of patients. Thus, our present study to address whether anthracycline is still required in addition to TC chemotherapy for successful treatment of Stage III/IV endometrial carcinoma is quite important.

There are minor drawbacks to our study; it was a retrospective analysis of TC and TEC and thus was not the ideal, which is a prospective randomized study. In addition, the treatment data were from two center hospitals for gynecologic malignancies; the Osaka University Hospital, in which TEC regimen was conducted, and the Osaka Medical Center for Cancer and Cardiovascular Diseases, in which TC was used. Both hospitals shared common practices for 

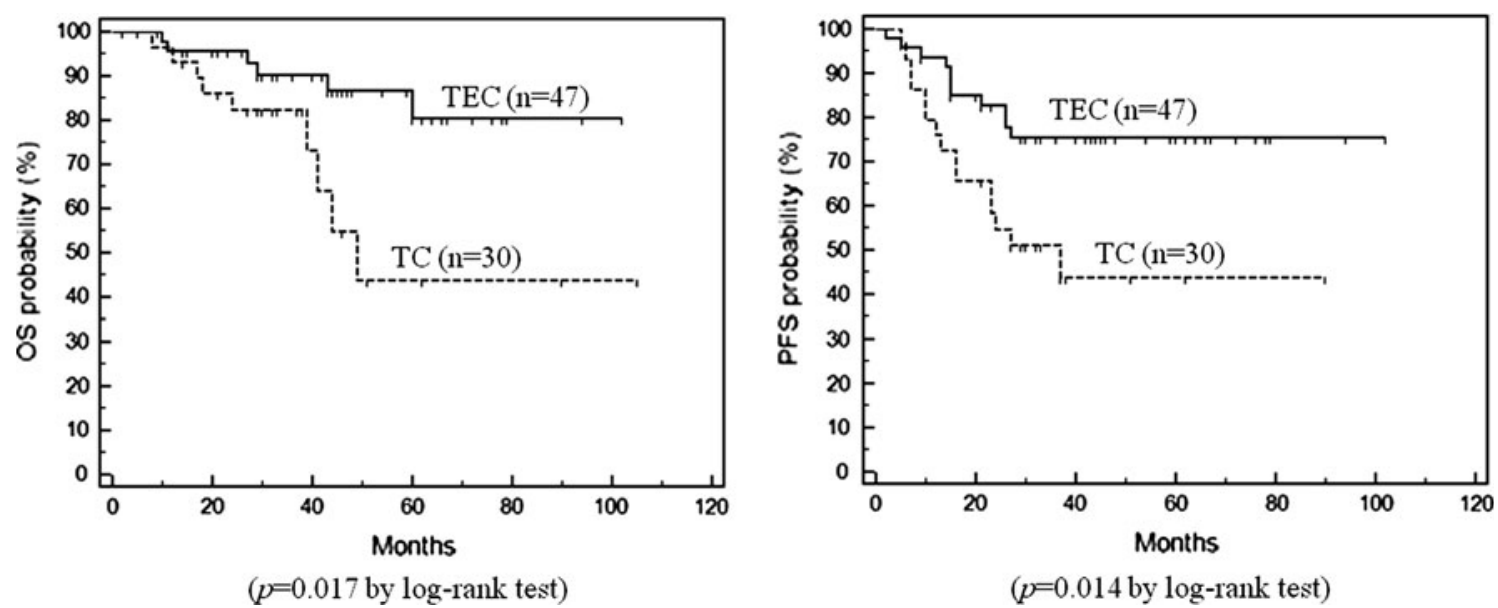

Fig. 2 PFS and OS of TEC and TC groups for adjuvant therapy (Stage III/IV). Both PFS and OS were significantly better in the TEC group than the TC group ( $p=0.017$ and $p=0.014$ by the log-rank test, respectively). Solid line TEC group. Broken line TC group

Table 4 Adverse effects of TEC and TC therapies

\begin{tabular}{llll}
\hline Toxicities & TEC & TC & $p$ value \\
\hline Hematological (Grade 4) & $67 / 115(57 \%)$ & $32 / 72(44 \%)$ & 0.065 \\
$\begin{array}{l}\text { Non-hematological } \\
\quad \text { Grades 3, 4) }\end{array}$ & $4 / 115(3 \%)$ & $4 / 72(6 \%)$ & 0.49 \\
\hline
\end{tabular}

Grade of adverse effects was based on the National Cancer Institute's Common Toxicity Criteria (version 2.0)

surgery, chemotherapy indications and treatment procedures. We believe that typical types of bias derived from a retrospective study were minimized as much as possible. The background features of the patients compared in the current study were not significantly different between the TC and TEC groups.

Comparison of the adverse effects of the TEC and TC chemotherapies showed that hematological toxicity, especially neutropenia, tended to be more frequently observed in the TEC group than in the TC group $(p=0.065)$, however the neutropenia was tolerable when given G-CSF support. Non-hematological toxicity was observed similarly in the TEC and TC groups $(p=0.49)$. These toxicities should always be taken into consideration as part of the effectiveness of any chemotherapy.

Our previous study showed that significant survival improvement was not demonstrated using TEC compared to radiation as an adjuvant therapy in Stage I/II cases (submitted), and the positive effects of TEC and TC therapy on survival were not significantly different in the present study, indicating that TC therapy may take the place of an adjuvant therapy for Stage I/II endometrial carcinoma.

Finding similar survival effects for TC and TEC in the most advanced and intractable unresectable cases and recurrent cases ( $p=0.63$ for OS and $p=0.55$ for PFS, by the log-rank test) implied that TC may be used as a remission induction chemotherapy due to the abjectly poor prog- nosis of those cases and the lower rate of severe side effects for TC therapy than for TEC. The tendency for improved survival in recurrent cases following TC therapy $(p=0.32$ and $p=0.22$ for PFS and OS, respectively) compared to TEC especially suggested that TC may be appropriate for recurrent cases, too. On the other hand, in advanced unresectable cases, TEC therapy tended to be more effective than TC ( $p=0.17$ for PFS). For these cases, although the TEC may improve PFS, it will require G-CSF administration for its associated severe neutropenia. Alternatively, TC may be proposed because, even though it is marginally less effective than TEC, it is more tolerable.

In the completely resected Stage III/IV cases, TEC therapy was demonstrated to provide significantly better PFS than TC ( $p=0.017$ by the log-rank test, Hazard Ratio: 0.3838 ; 95\% CI: $0.1709-0.8623$ ) and OS ( $p=0.014$ by the log-rank test, Hazard Ratio: 0.3108 ; 95\% CI: 0.1048-0.9214) with a median follow-up period of 38 months (2-105 months). The reasons for these epic results are uncertain as yet, although the synergistic or additive effect of a third drug mechanism is anticipated to be the cause. The fact that TEC was much more effective, especially for the completely resected cases in Stage III/IV which were at high risk of recurrence, may imply that the TEC regimen might play a role in not only attacking the carcinoma cells forming the tumor mass but also in killing remaining carcinoma stem cells and metastases elsewhere, such as in the bone marrow, after the complete resection of the tumor mass.

In this study, we proposed to question the apparent increasing application of TC regimen to all forms of endometrial carcinoma with an indication for chemotherapy. Based on our results, TEC should be offered as the preferred adjuvant therapy to Stage III/IV patients, after complete surgical resection of their tumors. TEC may also be effective as a remission induction therapy for patients in 
advanced stages with unresectable diseases. On the other hand, the TC regimen may now be considered for listing as a standard treatment useful for recurrent cases as a remission induction therapy having minimal side effects, and for patients in Stage I/II with risk factors requiring an adjuvant therapy. We firmly believe that GOG \#209 and JGOG \#2043 studies will provide strong evidence supporting our findings and our proposals.

Details of ethics approval All patients provided written informed consent before the treatment commenced. This study was approved by the Institutional Review Board of Osaka Medical Center for Cancer and Cardiovascular Diseases (\#1006145029, June 14, 2010) and the Ethics Committee of Osaka University Graduate School of Medicine (\#117, July 2, 2001).

Acknowledgments We would like to thank Dr. G. S. Buzard, CDCP, for his constructive editing of our manuscript. We are also grateful to Ms. S. Sugiyama and Ms. K. Nakano for their dedicated and excellent bioinformatics work extracting patient data from our medical records.

Conflict of interest The authors have no conflicts of interest to declare.

Open Access This article is distributed under the terms of the Creative Commons Attribution Noncommercial License which permits any noncommercial use, distribution, and reproduction in any medium, provided the original author(s) and source are credited.

\section{References}

1. DiSaia PJ, Creasman WT (2002) Clinical Gynecologic Oncology, 6th edn. Mosby, St. Louis

2. Berek JS (2002) Novak's Gynecology, 13th edn. William and Wilkins, Baltimore

3. Randall ME, Filiaci VL, Muss H, Spirtos NM, Mannel RS, Fowler J, Thigpen JT, Benda JA, Gynecologic Oncology Group Study (2006) Randomized phase III trial of whole-abdominal irradiation versus doxorubicin and cisplatin chemotherapy in advanced endometrial carcinoma: A Gynecologic Oncology Group Study. J Clin Oncol 24:36-44

4. Muss HB (1994) Chemotherapy of metastatic endometrial cancer. Semin Oncol 21:107-113

5. Thigpen JT, Brady MF, Homesley HD, Malfetano J, DuBeshter B, Burger RA, Liao S (2004) Phase III trial of doxorubicin with or without cisplatin in advanced endometrial carcinoma: a gynecologic oncology group study. J Clin Oncol 22:3902-3908

6. Ball HG, Blessing JA, Lentz SS, Mutch DG (1996) A phase II trial of paclitaxel in patients with advanced or recurrent adenocarcinoma of the endometrium: a Gynecologic Oncology Group study. Gynecol Oncol 62:278-281

7. Lissoni A, Zanetta G, Losa G, Gabriele A, Parma G, Mangioni C (1996) Phase II study of paclitaxel as salvage treatment in advanced endometrial cancer. Ann Oncol 7:861-863
8. Fleming GF, Brunetto VL, Cella D, Look KY, Reid GC, Munkarah AR, Kline R, Burger RA, Goodman A, Burks RT (2004) Phase III trial of doxorubicin plus cisplatin with or without paclitaxel plus filgrastim in advanced endometrial carcinoma: a Gynecologic Oncology Group Study. J Clin Oncol 22:2159-2166

9. Lissoni A, Gabriele A, Gorga G, Tumolo S, Landoni F, Mangioni C, Sessa C (1997) Cisplatin-, epirubicin- and paclitaxelcontaining chemotherapy in uterine adenocarcinoma. Ann Oncol 8:969-972

10. Papadimitriou CA, Bafaloukos D, Bozas G, Kalofonos H, Kosmidis P, Aravantinos G, Fountzilas G, Dimopoulos MA, Hellenic Co-operative Oncology Group (2008) Paclitaxel, epirubicin, and carboplatin in advanced or recurrent endometrial carcinoma: a Hellenic Co-operative Oncology Group (HeCOG) study. Gynecol Oncol 110:87-92

11. Sovak MA, DuPont J, Hensley ML, Ishill N, Gerst S, Abu-Rustum N, Anderson S, Barakat R, Konner J, Poyner E, Sabbatini P, Spriggs DR, Aghajanian C (2007) Paclitaxel and carboplatin in the treatment of advanced or recurrent endometrial cancer: a large retrospective study. Int J Gynecol Cancer 17:197-203

12. Pectasides D, Xiros N, Papaxoinis G, Pectasides E, Sykiotis C, Koumarianou A, Psyrri A, Gaglia A, Kassanos D, Gouveris P, Panayiotidis J, Fountzilas G, Economopoulos T (2008) Carboplatin and paclitaxel in advanced or metastatic endometrial cancer. Gynecol Oncol 109:250-254

13. Vandenput I, Vergote I, Leunen K, Berteloot P, Neven P, Amant F (2009) Leuven dose-dense paclitaxel/carboplatin regimen in patients with primary advanced or recurrent endometrial carcinoma. Int J Gynecol Cancer 19:1147-1151

14. Ueno Y, Enomoto T, Otsuki Y, Sugita N, Nakashima R, Yoshino K, Kuragaki C, Ueda Y, Aki T, Ikegami H, Yamazaki M, Ito K, Nagamatsu M, Nishizaki T, Asada M, Kameda T, Wakimoto A, Mizutani T, Yamada T, Murata Y (2006) Prognostic significance of p53 mutation in suboptimally resected advanced ovarian carcinoma treated with the combination chemotherapy of paclitaxel and carboplatin. Cancer Lett 241:289-300

15. World Health Organization (1979) Handbook of Reporting Results of Cancer Treatment No. 48. WHO Offset Publication, Geneva

16. Pectasides D, Xiros N, Papaxoinis G, Pectasides E, Sykiotis C, Koumarianou A, Psyrri A, Gaglia A, Kassanos D, Gouveris P, Panayiotidis J, Fountzilas G, Economopoulos T (2008) Carboplatin and paclitaxel in advanced or metastatic endometrial cancer. Gynecol Oncol 109:250-254

17. du Bois A, Lück HJ, Meier W, Adams HP, Möbus V, Costa S, Bauknecht T, Richter B, Warm M, Schröder W, Olbricht S, Nitz U, Jackisch C, Emons G, Wagner U, Kuhn W, Pfisterer J, Arbeitsgemeinschaft Gynäkologische Onkologie Ovarian Cancer Study Group (2003) A randomized clinical trial of cisplatin/paclitaxel versus carboplatin/paclitaxel as first-line treatment of ovarian cancer. J Natl Cancer Inst 95:1320-1329

18. Egawa-Takata T, Ueda Y, Kuragaki C, Miyake T, Miyatake T, Fujita M, Yoshino K, Nakashima R, Okazawa M, Tsutsui T, Morishige K, Kimura T, Yamasaki M, Nishizaki T, Nagamatsu M, Ito K, Asada M, Ogita K, Wakimoto A, Yamamoto T, Nishio Y, Enomoto T (2011) Chemotherapy for endometrial carcinoma (GOGO-EM1 study): TEC (paclitaxel, epirubicin, and carboplatin) is an effective remission-induction and adjuvant therapy. Cancer Chemother Pharmacol 68:1603-1610 